\title{
Theoretical Insights into Proton-Coupled Electron Transfer from a Photoreduced ZnO Nanocrystal to an Organic Radical
}

\author{
Soumya Ghosh, ${ }^{\dagger}$ Janelle Castillo-Lora, $^{\dagger}$ Alexander V. Soudackov, ${ }^{\dagger}$ James M. Mayer, $^{\ddagger}$
} and Sharon Hammes-Schiffer* ${ }^{*} \dagger$

\author{
${ }^{\dagger}$ Department of Chemistry, University of Illinois at Urbana-Champaign, 600 South Mathews Avenue, Urbana, Illinois 61801, United \\ States \\ ${ }^{\ddagger}$ Department of Chemistry, Yale University, New Haven, Connecticut 06520, United States
}

\section{Supporting Information}

ABSTRACT: Proton-coupled electron transfer (PCET) at metal-oxide nanoparticle interfaces plays a critical role in many photocatalytic reactions and energy conversion processes. Recent experimental studies have shown that photoreduced $\mathrm{ZnO}$ nanocrystals react by PCET with organic hydrogen atom acceptors such as the nitroxyl radical TEMPO. Herein, the interfacial PCET rate constant is calculated in the framework of vibronically nonadiabatic PCET theory, which treats the electrons and transferring proton quantum mechanically. The input quantities to the PCET rate constant, including the electronic couplings, are calculated with density functional theory. The computed interfacial PCET rate constant is consistent with the experimentally measured value for this system,

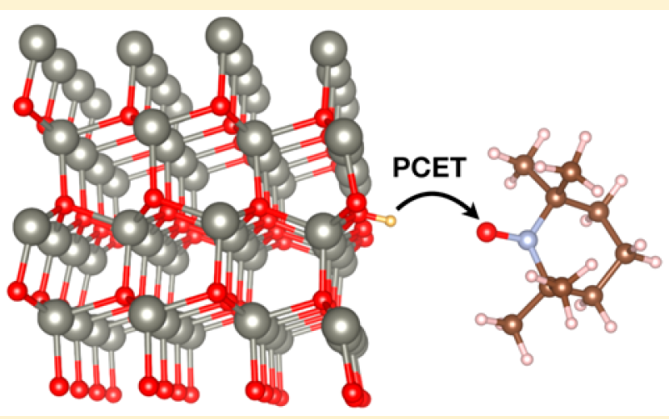
providing validation for this PCET theory. In this model, the electron transfers from the conduction band of the $\mathrm{ZnO}$ nanocrystal to TEMPO concertedly with proton transfer from a surface oxygen of the $\mathrm{ZnO}$ nanocrystal to the oxygen of TEMPO. Moreover, the proton tunneling at the interface is gated by the relatively lowfrequency proton donor-acceptor motion between the TEMPO radical and the $\mathrm{ZnO}$ nanocrystal. The $\mathrm{ZnO}$ nanocrystal and TEMPO are found to contribute similar amounts to the inner-sphere reorganization energy, implicating structural reorganization at the nanocrystal surface. These fundamental mechanistic insights may guide the design of metal-oxide nanocatalysts for a wide range of energy conversion processes.

KEYWORDS: Proton-coupled electron transfer, nanocrystal, nanoparticle, proton tunneling, density functional theory, zinc oxide

$\mathrm{P}$ roton-coupled electron transfer (PCET) at semiconductorsolution interfaces plays a pivotal role in a wide range of energy conversion processes and photocatalytic reactions, especially in photochemical water splitting. ${ }^{1-7}$ While various types of metal oxide nanoparticles have been shown to exhibit significant photocatalytic activity, many studies focus solely on the electron transfer reactions at these interfaces ${ }^{8-11}$ and neglect the essential role of the protons. ${ }^{12,13}$ Thus, a fundamental mechanistic understanding of interfacial PCET for metal oxide nanoparticles is still lacking. Recently, photoreduced colloidal $\mathrm{ZnO}$ nanocrystals (NCs) have been shown to react by PCET with organic hydrogen atom acceptors such as the nitroxyl radical TEMPO. ${ }^{14}$ In these experiments, the photoreduced $\mathrm{ZnO}$ nanocrystals were prepared using ultraviolet irradiation to form electron-hole pairs, followed by rapid quenching of the holes through the oxidation of ethanol to acetaldehyde. This procedure produces the photoreduced NCs denoted as $\mathrm{ZnO}: e_{\mathrm{CB}}^{-} / \mathrm{H}^{+}$, where the subscript $\mathrm{CB}$ indicates that the reducing electrons occupy delocalized orbitals in the conduction band (CB). The locations of the protons were not established by these experiments, but previous experimental and theoretical studies on reduced bulk $\mathrm{ZnO}$ suggest that protons can be both at the surface and intercalated. ${ }^{5,16}$ The reaction of $\mathrm{ZnO}: e_{\mathrm{CB}}^{-} / \mathrm{H}^{+}$with TEMPO transfers an electron and a proton to oxidize the $\mathrm{NC}$ and form the reduced and protonated TEMPOH. ${ }^{14}$

In this Letter, we calculate the rate constant for this net hydrogen atom transfer from a photoreduced $\mathrm{ZnO} \mathrm{NC}$ to a TEMPO molecule:

$$
\mathrm{ZnO}: e_{\mathrm{CB}}^{-} / \mathrm{H}^{+}+\mathrm{TEMPO} \cdot \stackrel{k_{2}}{\rightarrow} \mathrm{ZnO}+\mathrm{TEMPOH}
$$

within the framework of nonadiabatic PCET theory. The kinetics of this reaction in toluene have been examined both by flash photolysis with transient absorption ${ }^{14}$ and by stoppedflow mixing. ${ }^{17}$ The latter studies have shown that the dependence of the rate on the average concentration of electrons in the NCs is relatively weak. ${ }^{19}$ For simplicity, the calculations herein consider only reduction by a single $\mathrm{H}$ atom equivalent, and the computed rate constant is compared to the results from flash photolysis studies with an average number of electrons per NC that is less than one. ${ }^{14}$ These experimental

Received: June 29, 2017

Revised: August 24, 2017

Published: August 28, 2017 


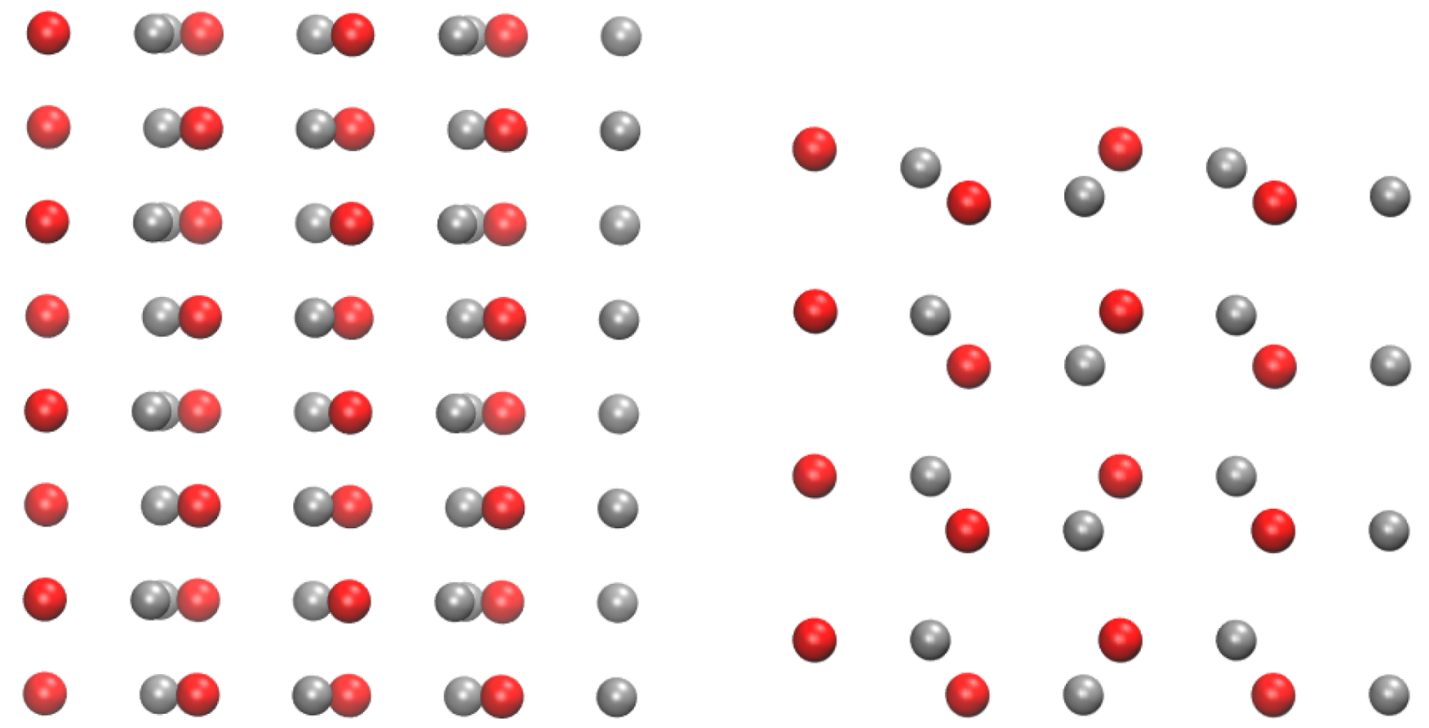

Figure 1. Top view (left) and side view (right) of the optimized structures of the nonpolar $10 \overline{1} 0 \mathrm{ZnO}$ surface, where the $\mathrm{Zn}$ atoms are gray and the $\mathrm{O}$ atoms are red.

studies produced a second-order rate constant for the initial portion of the reaction of $\sim 10^{3} \mathrm{M}^{-1} \mathrm{~s}^{-1}$.

These flash photolysis experiments can be described by rapid, reversible formation of a hydrogen-bonded adduct with equilibrium constant $K_{\text {eq }}$, followed by a first-order PCET reaction with rate constant $k_{\mathrm{PCET}}$. In this case, the overall mechanism can be written as

$$
\begin{gathered}
\mathrm{ZnO}: e_{\mathrm{CB}}^{-} / \mathrm{H}^{+}+\text {TEMPO } \stackrel{K_{\mathrm{eq}}}{\rightleftarrows}\left[\mathrm{ZnO}: e_{\mathrm{CB}}^{-} / \mathrm{H}^{+} \cdots \mathrm{TEMPO} \cdot\right] \\
{\left[\mathrm{ZnO}: e_{\mathrm{CB}}^{-} / \mathrm{H}^{+} \cdots \mathrm{TEMPO} \cdot\right] \stackrel{k_{\mathrm{PCET}}}{\longrightarrow}[\mathrm{ZnO} \cdots \mathrm{TEMPOH}]}
\end{gathered}
$$

followed by fast dissociation of the hydrogen-bonded product. Under steady-state conditions, the equilibrium associated with forming the hydrogen-bonded complex occurs on a much faster time scale than the subsequent PCET reaction. In this case, the second-order rate constant is simply

$$
k_{2}=K_{\mathrm{eq}} k_{\text {PCET }}
$$

This kinetic scheme is based on the assumption that PCET reactions to different hydrogen-bonded TEMPO molecules are independent and are described by first-order kinetics in [TEMPO] and in the concentration of electrons in the conduction band of the NCs. The photochemical experiments were conducted in the presence of a large excess of TEMPO and are consistent with this scheme. Our goal is to compute the interfacial PCET rate constant $k_{\mathrm{PCET}}$ using first-principles calculations for comparison to the experimental value and to elucidate the fundamental PCET mechanism. The key issues that are probed include the roles of hydrogen tunneling and the associated low-frequency gating mode as well as structural reorganization within the $\mathrm{ZnO} \mathrm{NC}$.

The reaction system consists of a model for the $\mathrm{ZnO}$ reactive surface and the TEMPO molecule. The $\mathrm{ZnO}$ reactive surface is assumed to be the nonpolar $10 \overline{1} 0$ surface, represented as a periodic slab, as depicted in Figure 1, with vacuum above and below it. The choice of the nonpolar surface over the polar oxygen-terminated surface was mainly dictated by the strong affinity of the polar surface toward hydrogen. Previous studies have shown that in the presence of hydrogen, the polar surface forms a stable monolayer, in contrast to the nonpolar surface. ${ }^{16}$ Hence, the removal of a hydrogen atom is expected to be thermodynamically less favorable for the polar surface than for the nonpolar surface. Details about the construction of the model for this surface are provided in the Supporting Information. The climbing image nudged elastic band (CI$\mathrm{NEB}$ ) method $^{18}$ was used to generate the reaction path for net hydrogen atom transfer from the $\mathrm{ZnO} \mathrm{NC}$ to TEMPO using density functional theory (DFT) with the PBE functional. ${ }^{19-21}$ The PBE functional was used to optimize geometries because it has been found to describe the structure of bulk $\mathrm{ZnO}$ reasonably accurately. ${ }^{22}$ However, we found that this functional is inadequate for computing accurate reaction energies, as will be discussed below. Therefore, a hybrid functional, PBE0-TCLRC, $^{23}$ with a smaller basis $\operatorname{set}^{24}$ was used to calculate the reaction energies, reorganization energies, and other energetic properties. Additional computational details are given in the Supporting Information. The corresponding transition state geometry of the reaction system is depicted in Figure 2.

For simplicity, the dodecylamine (DDA) capping ligands that were present in the experimental $\mathrm{ZnO}$ NCs were not included in the model, so any interaction between those ligands and the surface protons would not be captured by this treatment. It seems unlikely that the surface protons are present as dodecylammonium ions $\left(\mathrm{RNH}_{3}^{+}\right)$. Preliminary studies with trioctylphosphine oxide-capped $\mathrm{ZnO}$ showed similar but slightly slower reactivity with TEMPO, suggesting that the capping ligands do not play a major role (Figure S1). In other experiments, the addition of electrons to $\mathrm{ZnO} N C s$ was greatly enhanced by added $\mathrm{RNH}_{3}{ }^{+}$but not by ${ }^{n} \mathrm{Bu}_{4} \mathrm{~N}^{+}$, indicating that the acidic ammonium ion simply transfers a proton to the NC to stabilize the charge of the electron and further suggesting that the capping ligand plays only a subsidiary role. ${ }^{25,26}$

A vibronically nonadiabatic PCET rate constant expres$\operatorname{sion}^{27-30}$ has been derived by treating the electrons and transferring proton quantum mechanically. In this theory, which may be viewed as an extension of Marcus theory for electron transfer, ${ }^{31-33}$ the PCET reaction is described in terms of nonadiabatic transitions between electron-proton vibronic states. This PCET theory can be extended to a semiconductor- 


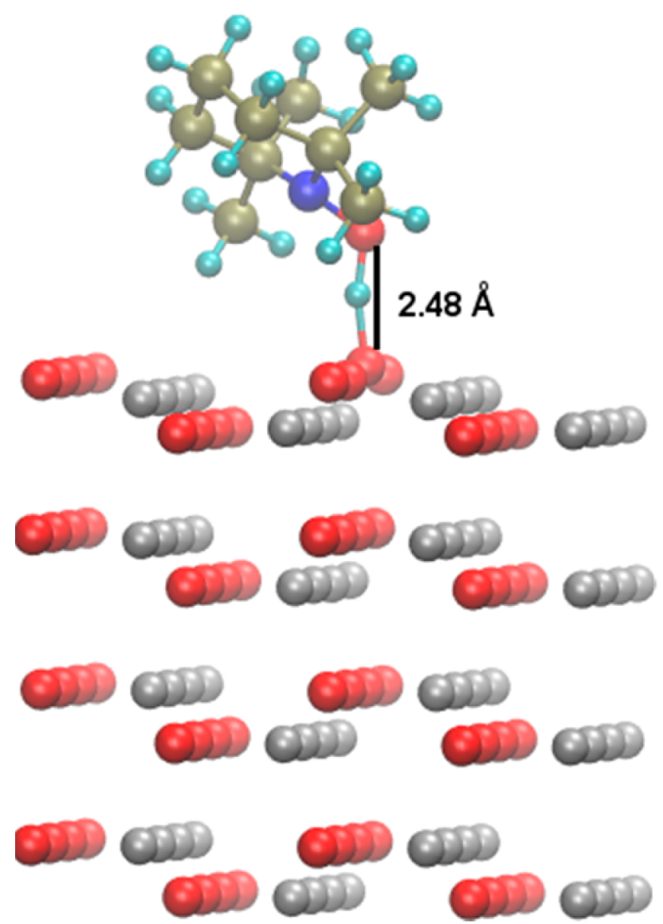

Figure 2. Approximate transition state geometry for the net hydrogen atom transfer from $\mathrm{ZnO}: e_{\mathrm{CB}}^{-} / \mathrm{H}^{+}$to TEMPO. The color scheme is as follows: $\mathrm{Zn}$ (gray), O (red), N (blue), C (green), H (cyan). The proton donor-acceptor distance (in $\AA$ ) is shown in black.

liquid interface by considering the energy levels in the semiconductor. The resulting rate constant can be expressed as:

$$
k_{\mathrm{PCET}}=\int_{0}^{\infty} k(R) P(R) d R
$$

where $R$ is the proton donor-acceptor distance (i.e., the distance between a surface oxygen on $\mathrm{ZnO}$ and the oxygen of TEMPO), and $P(R)$ is the probability distribution function for this distance. The PCET rate constant at a particular $R$ is given by:

$$
k(R)=\frac{1}{\hbar} \sqrt{\frac{\pi}{k_{\mathrm{B}} T \lambda}} \sum_{\mu, \nu} P_{\mu} S_{\mu \nu}(R)^{2} \int_{-\infty}^{\varepsilon_{\mathrm{F}}} \mathrm{d} \varepsilon|V(\varepsilon)|^{2} \exp \left[-\frac{\Delta G_{\mu \nu}^{\ddagger}(\varepsilon, R)}{k_{\mathrm{B}} T}\right]
$$

In eq 5 , the double summation is over all pairs of reactant $(\mu)$ and product $(\nu)$ electron-proton vibronic states, $P_{\mu}$ is the Boltzmann population of the reactant state $\mu, \lambda$ is the total reorganization energy, and $S_{\mu \nu}$ is the overlap integral between the reactant and product proton vibrational wave functions. The integral is over the occupied energy levels in the semiconductor, where $\varepsilon_{\mathrm{F}}$ is the Fermi energy and $|V(\varepsilon)|^{2}$ describes the density of states weighted by the square of the electronic coupling: $|V(\varepsilon)|^{2}=\int \mathrm{d} \mathbf{k}\left|V_{k}\right|^{2} \delta(\varepsilon(\mathbf{k})-\varepsilon)$. In eq 5, $\Delta G^{\ddagger}{ }_{\mu \nu}(\varepsilon, R)$ is the free energy barrier for the pair of reactant and product vibronic states $\mu / \nu$ and can be expressed as:

$$
\Delta G_{\mu \nu}^{\ddagger}(\varepsilon, R)=\frac{\left[\Delta G_{\mu \nu}(\varepsilon, R)+\lambda\right]^{2}}{4 \lambda}
$$

where $\Delta G_{\mu \nu}(\varepsilon, R)$ is the reaction free energy for the reactant and product vibronic states, $\mu$ and $\nu$, respectively. The explicit form of the reaction free energy, as well as the numerical procedure for calculating the integral in eq 5 , is given in the Supporting Information.
As discussed earlier, the initial photoexcitation generates an exciton that is rapidly trapped by oxidation of surface ethanol in ca. 15 ps. ${ }^{34}$ Experimentally, electrons in the $\mathrm{CB}$ are needed to observe PCET reactivity, and what is being measured in the kinetic studies is the decay of the average concentration of $\mathrm{CB}$ electrons. For the calculation of the rate constant, all of the occupied molecular orbitals (MOs) are included in eq 5 to account for electron transfer from the valence band (VB) as well as the $C B$. When the electron transfers from the $V B$, relaxation of an electron from the $\mathrm{CB}$ to the $\mathrm{VB}$ is assumed to be fast compared to the PCET reaction. Thus, electron transfer from either the $\mathrm{VB}$ or the $\mathrm{CB}$ leads to the experimentally observed decrease in the average concentration of $\mathrm{CB}$ electrons. However, as described below, the contributions of the $\mathrm{VB}$ orbitals were found to be negligible because of the substantial energy gap between the VB and the $\mathrm{CB}$.

The electronic coupling is calculated by partitioning the total system into the donor, $\mathrm{ZnO}: e_{\mathrm{CB}}^{-}$, and the acceptor, $\mathrm{TEMPOH}^{+}$. The couplings between the lowest unoccupied molecular orbital (LUMO) of the acceptor and all of the occupied MOs on the donor were extracted from electronic structure calculations following the procedure described in ref 35 . At the transition state geometry, the computed couplings are similar if the partitioning scheme defines the proton as part of the donor rather than the acceptor. Starting at the transition state geometry obtained with the CI-NEB method (Figure 2), the coupling calculations were performed at different values of $R$ by translating the two rigid fragments along the vector connecting the proton donor and acceptor atoms and evaluating the electronic couplings at each distance. As discussed in the Supporting Information, the couplings were calculated for both partially frozen and fully relaxed $\mathrm{ZnO}$ structures. Figures $\mathrm{S} 6$ and S7 illustrate that the highest occupied MO (HOMO) of the donor is delocalized over the entire NC for both types of calculations. Note that these calculations do not require the definition of a donor center but rather require the explicit calculation of couplings between the occupied MOs of the NC and the unoccupied acceptor MO. Additional details about the coupling calculations are provided in the Supporting Information.

We found that the PCET rate constant is the same to within three significant figures when the rate constant is computed with and without the contributions from the VB orbitals. Figure 3 illustrates the dependence of the electronic coupling on the

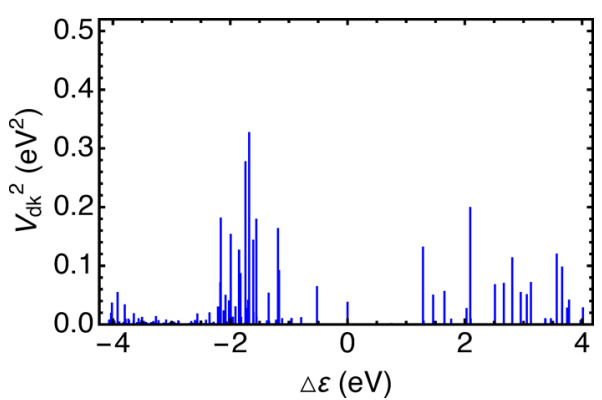

Figure 3. Plot of the square of the electronic coupling vs the energies of the MOs of the donor fragment $\left(\mathrm{ZnO}: e_{\mathrm{CB}}^{-}\right)$at the dominant proton donor-acceptor distance $R=2.30 \AA$ for the partially frozen $\mathrm{ZnO}$ structure. All of the MO energies are relative to the energy of the NC HOMO (bottom of the conduction band), and positive MO energies correspond to unoccupied orbitals that were not included in the calculation. 
donor MO energies at the dominant proton donor-acceptor distance. Although the electronic couplings may be similar or even larger for the $\mathrm{VB}$ orbitals compared to the $\mathrm{NC} \mathrm{HOMO}$ (i.e., the bottom of the $\mathrm{CB}$ ), the substantial energy gap results in only negligible contributions of the $\mathrm{VB}$ orbitals to the overall PCET rate constant. Note that the vibronically nonadiabatic rate constant expression in eq 5 requires the vibronic coupling (i.e., the product of the electronic coupling and the overlap integral between the proton vibrational wave functions) to be less than the thermal energy $k_{\mathrm{B}} T$, which is $0.025 \mathrm{eV}$ at $295 \mathrm{~K}$. For this system, the vibronic nonadiabaticity can be analyzed in terms of the product of the electronic coupling between the HOMO of the donor and the LUMO of the acceptor and the overlap integral for the ground vibronic states at the dominant and equilibrium proton donor-acceptor distances. For the partially frozen $\mathrm{ZnO}$ structures, the relevant vibronic coupling was $0.021 \mathrm{eV}$ at the dominant proton donor-acceptor distance $(R=2.30 \AA)$ and $4.4 \times 10^{-7} \mathrm{eV}$ at the equilibrium distance $(R$ $=2.74 \AA$ ) . For the fully relaxed $\mathrm{ZnO}$ structures, the relevant vibronic coupling was $0.0028 \mathrm{eV}$ at the dominant proton donor-acceptor distance $(R=2.45 \AA)$ and $7.3 \times 10^{-6} \mathrm{eV}$ at the equilibrium distance $(R=2.74 \AA$ ). These values justify the use of the vibronically nonadiabatic rate constant expression for this system at $295 \mathrm{~K}$.

The free energy difference $\Delta G_{\mu \nu}(\varepsilon, R)$ includes the intrinsic energy difference $\Delta E$ between the reactant and product as well as the energy difference between the reactant and product proton vibrational energy levels. On the basis of eq 2 , the reactant is $\mathrm{ZnO}: e_{\mathrm{CB}}^{-} / \mathrm{H}^{+} \ldots$ TEMPO, and the product is $\mathrm{ZnO} \cdots$ TEMPOH. The reactant and product energies are approximated as the sum of the individual fragment energies, thereby assuming that the hydrogen-bonding energy is the same in both the reactant and product. Thus, $\Delta E=\left(E_{\mathrm{TEMPOH}}-E_{\mathrm{TEMPO}}\right)-$ $\left(E_{\mathrm{ZnO}: e_{\mathrm{CB}}^{-} / \mathrm{H}^{+}}-E_{\mathrm{ZnO}}\right)$, which was computed to be $-0.339 \mathrm{eV}$, indicating that this reaction is highly exothermic. This reaction energy can be decomposed into the $\mathrm{O}-\mathrm{H}$ hydrogen dissociation energies of TEMPOH and $\mathrm{ZnO}: e_{\mathrm{CB}}^{-} / \mathrm{H}^{+}$, which were computed to be $2.99 \mathrm{eV}(69.0 \mathrm{kcal} / \mathrm{mol})$ and $2.65 \mathrm{eV}$ $(61.2 \mathrm{kcal} / \mathrm{mol})$, respectively. The computed dissociation energy of TEMPOH is in qualitative agreement with the experimental data $\left(\sim 65 \mathrm{kcal} / \mathrm{mol}\right.$ in nonpolar solvent). ${ }^{36}$ Experimentally, the equilibrium in eq 2 is well-shifted to products because the treatment of unreduced $\mathrm{ZnO}(0.015 \mathrm{mM})$ with excess TEMPOH $(1 \mathrm{mM})$ does not result in any measurable formation of $\mathrm{ZnO}: e_{\mathrm{CB}}^{-} / \mathrm{H}^{+}$, as shown in Figure 4, indicating that the effective $\mathrm{ZnO}-\mathrm{H}$ bond dissociation free energy (BDFE) is less than that of TEMPO. A more quantitative estimate can be obtained with two assumptions. First, the $\mathrm{O}-\mathrm{H}$ bonds are similar between the DDA-capped $\mathrm{ZnO} \mathrm{NCs}$ studied experimentally and $\mathrm{ZnO}$ electrodes facing aqueous solutions, determined based on such BDFEs being fairly solvent independent. ${ }^{36}$ Second, taking the typical measured $\mathrm{ZnO}$ conduction band edge energy of ca. $-0.3 \mathrm{~V}$ versus $\mathrm{NHE}^{37}$ as the reduction potential for the $1 e^{-}+1 \mathrm{H}^{+}$ reaction (a nonstandard assumption; ${ }^{38}$ this connection between measured band energies and surface $\mathrm{O}-\mathrm{H}$ BDFEs will be developed in a future publication $)^{39}$ gives an estimated effective BDFE of $\sim 50 \mathrm{kcal} \mathrm{mol}^{-1} \cdot{ }^{39}$ Given the assumptions made in this estimate, as well as limitations in using DFT to calculate dissociation energies, the calculated values are in reasonable agreement with the experimentally based estimates.

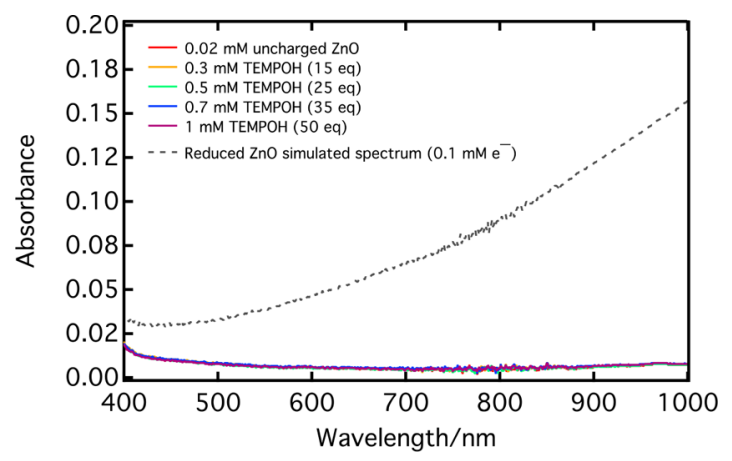

Figure 4. Optical spectra tracking the addition of TEMPOH to unreduced $\mathrm{ZnO}$. To an initial solution containing $0.02 \mathrm{mM}$ of uncharged $\mathrm{ZnO}$ were added 4 aliquots of TEMPOH. The final solution, which is composed of $1 \mathrm{mM}$ of TEMPOH and $0.015 \mathrm{mM}$ of uncharged $\mathrm{ZnO}$, did not show any spectroscopic changes that would indicate reduction of $\mathrm{ZnO}$ by TEMPOH. An example spectrum indicating reduction of $\mathrm{ZnO}$ is shown by the gray dashed optical profile extending from the visible to the near-infrared region.

The total reorganization energy is the sum of the outersphere (solvent) and inner-sphere (solute) reorganization energies. The solvent reorganization energy is assumed to be negligible compared to the inner-sphere reorganization energy because the reaction occurs in the relatively nonpolar solvent toluene, and the net hydrogen atom transfer does not change the overall charge on the donor or acceptor fragments. As a result, the total reorganization energy is equated to the innersphere reorganization energy, which is calculated with a variant of the four-point method. ${ }^{4,41}$ (In the absence of large solvent reorganization energy, the low-frequency modes in the $\mathrm{NC}$ and TEMPO are assumed to be sufficient to justify the use of the golden rule PCET rate constant expression corresponding to the high temperature limit.) In this case, the energies of the equilibrium and nonequilibrium geometries of the isolated fragments are combined to compute the inner-sphere reorganization energy as follows:

$$
\begin{aligned}
\lambda_{\text {in }}= & \frac{1}{2}\left\{\left[E_{\mathrm{TEMPO}}(\mathrm{TEMPOH})+E_{\mathrm{ZnO}: e_{\mathrm{CB}}^{-} / \mathrm{H}^{+}}(\mathrm{ZnO})\right.\right. \\
& \left.-E_{\mathrm{TEMPO}}(\mathrm{TEMPO})-E_{\mathrm{ZnO}: e_{\mathrm{CB}}^{-} / \mathrm{H}^{+}}\left(\mathrm{ZnO}: e_{\mathrm{CB}}^{-} / \mathrm{H}^{+}\right)\right] \\
+ & {\left[E_{\mathrm{TEMPOH}}(\mathrm{TEMPO})+E_{\mathrm{ZnO}}\left(\mathrm{ZnO}: e_{\mathrm{CB}}^{-} / \mathrm{H}^{+}\right)\right.} \\
& \left.\left.-E_{\mathrm{TEMPOH}}(\mathrm{TEMPOH})-E_{\mathrm{ZnO}}(\mathrm{ZnO})\right]\right\}
\end{aligned}
$$

Here $E_{\mathrm{A}}(\mathrm{B})$ denotes the energy of state $\mathrm{A}$ at the optimized geometry of $\mathrm{B}$, where $\mathrm{A}$ and $\mathrm{B}$ can be TEMPO, TEMPOH, $\mathrm{ZnO}$, or $\mathrm{ZnO}: e_{\mathrm{CB}}^{-} / \mathrm{H}^{+}$, depending on the particular term in eq 7 . If $\mathrm{A}$ and $\mathrm{B}$ are the same, then this energy corresponds to the equilibrium energy of that species, but if they are different, then this energy corresponds to a nonequilibrium geometry. If $\mathrm{B}$ is protonated and $\mathrm{A}$ is not protonated, the proton on $\mathrm{B}$ is removed, while if $B$ is not protonated and $A$ is protonated, then the proton is optimized for state A while fixing all other atoms to the optimized geometry of B. Note that the inner-sphere reorganization energy is calculated for isolated species rather than for the hydrogen-bonded complex in eq 2, based on the reasonable assumption that it does not depend significantly on the distance between TEMPO and the $\mathrm{ZnO}$ NC. The innersphere reorganization energy was computed to be $1.6 \mathrm{eV}$. Analysis of the different components shows that both TEMPO and the $\mathrm{ZnO} \mathrm{NC}$ contribute similarly to this value. A 
comparison of the optimized geometries for $\mathrm{ZnO}$ and $\mathrm{ZnO}: e_{\mathrm{CB}}^{-} / \mathrm{H}^{+}$is depicted in Figure $\mathrm{S} 4$, suggesting that the structural differences occur predominantly at the surface, especially the $\mathrm{Zn}$ atoms around the $\mathrm{OH}$ group due to the breaking of this bond.

To calculate the proton vibrational states associated with the reactant and product, we used Morse potentials for the $\mathrm{OH}$ bonds of the $\mathrm{ZnO}: e_{\mathrm{CB}}^{-} / \mathrm{H}^{+}$and TEMPOH species, as depicted in Figure S5. The parameters for the Morse potentials were obtained as described in Table S1. The proton vibrational energy levels and wave functions were computed analytically, and the overlap integrals were calculated numerically. The proton donor-acceptor mode (i.e., the motion of TEMPO relative to the $\mathrm{ZnO}$ surface in the hydrogen-bonded complex $\left.\left[\mathrm{ZnO}: e_{\mathrm{CB}}^{-} / \mathrm{H}^{+} \ldots \mathrm{TEMPO} \cdot\right]\right)$ was approximated to be harmonic, and the corresponding force constant $\left(f_{\mathrm{R}}\right)$ was estimated numerically to be $67.38 \mathrm{kcal} \mathrm{mol}^{-1} \AA^{-2}$. As given in eq 4 , the overall rate constant for PCET $\left(k_{\mathrm{PCET}}\right)$ is evaluated numerically as the integral of the product of $P(R)$ and $k(R)$, which are shown in Figure 5.

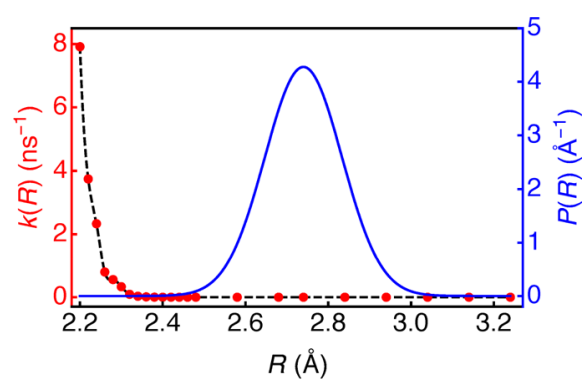

Figure 5. PCET rate constant, $k(R)$ (red points), and probability distribution function of the proton donor-acceptor distance, $P(R)$ (blue line), for PCET from $\mathrm{ZnO}: e_{\mathrm{CB}}^{-} / \mathrm{H}^{+}$to TEMPO calculated using the partially frozen $\mathrm{ZnO}$ slab. Here, $R$ is the distance between the proton donor oxygen on $\mathrm{ZnO}: e_{\mathrm{CB}}^{-} / \mathrm{H}^{+}$and the proton acceptor oxygen on TEMPO.

As illustrated in Figure 5, the overall rate constant is determined by a balance between the rate constant at each proton donor-acceptor distance $R$ and the probability of sampling that distance. The maximum of the Gaussian probability distribution function $P(R)$ is defined to be the donor-acceptor distance at the reactant equilibrium geometry, which is $2.74 \AA$, while the rate constant increases significantly as the proton tunneling distance decreases. Consequently, the dominant contribution to the overall PCET rate constant corresponds to the shorter distance of $R=2.30 \AA$ when the coupling is computed with the partially frozen $\mathrm{ZnO}$ slab and $R$ $=2.45 \AA$ when the coupling is computed with the fully relaxed $\mathrm{ZnO}$ slab. Although the probability $P(R)$ is very small at the dominant distance, the significantly larger value of the rate constant $k(R)$ leads to the maximum of the product of these two quantities. Analysis of the various contributions to the total rate constant at this distance shows that the dominant pair of reactant and product proton vibrational states corresponds to the ground states. A more detailed analysis of the contributions to the PCET rate constant is provided in Table S2.

The computed rate constant $k_{\mathrm{PCET}}$ using all of these input quantities is $2.5 \times 10^{3} \mathrm{~s}^{-1}$. This value utilizes the electronic coupling computed with the partially frozen $\mathrm{ZnO}$ slab to maintain consistency with the calculations of the other input quantities for the rate constant. The experimental value for the initial part of the reaction is ca. $10^{3} \mathrm{M}^{-1} \mathrm{~s}^{-1}$, as mentioned above. This is an approximate value because the kinetics showed some variability between batches of NCs and with different amounts of DDA capping ligands, and over longer time showed multiphasic behavior, which is a common feature of nanoparticle kinetics. For conversion from the experimental bimolecular rate constant to the computed first-order rate constant within the $\mathrm{ZnO}: e_{\mathrm{CB}}^{-} / \mathrm{H}^{+}$...TEMPO precursor complex, as given by eq 3 , an estimate of the $K_{\text {eq }}$ for TEMPO binding is needed.

One way to obtain a qualitative sense of this $K_{\mathrm{eq}}$ is to analyze the competition between TEMPO and the DDA capping ligands, some of which are quite weakly bound to the surface. ${ }^{42}$ The addition of excess TEMPO $(50 \mathrm{mM})$ to unreduced $\mathrm{ZnO}$ NCs $(0.2 \mathrm{mM}$ unreduced $\mathrm{ZnO}$ with $50 \mathrm{mM}$ DDA capping ligand from the synthesis) did not result in any observed binding or displacement of DDA ligands and showed no evidence of any PCET reactivity. To determine changes in the number of bound versus unbound DDA capping ligands, the MestReNova Fitting Function capability was used to fit the peak at $1.2-1.6 \mathrm{ppm}$ to two regimes of bound (down-field) and free and weakly bound (up-field) DDA capping ligands (Figure S3). Even with the addition of excess TEMPO, neither the number of free capping ligands nor the number of bound capping ligands changed. While this observation is not a direct measure of TEMPO binding, it suggests an upper limit for the preassociation $K_{\mathrm{eq}}$ of ca. $10 \mathrm{M}^{-1}$ based on the rough assumption that no more than about half the $\mathrm{ZnO}$ nanoparticles are bound to the $50 \mathrm{mM}$ TEMPO. Assuming $K_{\text {eq }} \approx 1$ $\mathrm{M}^{-1}$ based on previous estimates for nonbonded neutral electron-transfer couples, ${ }^{43}$ the computed PCET rate constant of $2.5 \times 10^{3} \mathrm{~s}^{-1}$ is consistent with the experimental secondorder rate constant of $\sim 10^{3} \mathrm{M}^{-1} \mathrm{~s}^{-1}$. This agreement suggests that the nonpolar $10 \overline{1} 0$ surface is a plausible candidate for the reactive surface at which PCET can occur between the photoreduced $\mathrm{ZnO} \mathrm{NC}$ and TEMPO and supports the validity of the vibronically nonadiabatic PCET model for describing this process.

In summary, the vibronically nonadiabatic PCET theory, in conjunction with first-principles calculations of the input quantities, is able to reproduce the experimentally measured rate constant for this interfacial PCET reaction. In addition to providing validation for the vibronically nonadiabatic PCET theory and the model used to represent the $\mathrm{ZnO} \mathrm{NC}$, these calculations provide insights into the fundamental PCET mechanism. According to this theory, the proton tunnels from a surface oxygen on the nonpolar surface of the $\mathrm{ZnO} \mathrm{NC}$ to the oxygen of TEMPO concertedly with electron transfer from the conduction band of the $\mathrm{ZnO} \mathrm{NC}$ to TEMPO. Furthermore, this reaction is gated by the relatively lowfrequency proton donor-acceptor $\mathrm{O}-\mathrm{O}$ vibrational motion at the hydrogen-bonding interface between the TEMPO radical and the $\mathrm{ZnO} \mathrm{NC}$. Another insight from the calculations is that the $\mathrm{ZnO} \mathrm{NC}$ and TEMPO contribute similar amounts to the inner-sphere reorganization energy, implying the significance of structural reorganization at the NC surface. These fundamental mechanistic insights may be valuable for designing metal oxide nanocatalysts for energy conversion processes. 


\section{ASSOCIATED CONTENT}

\section{S Supporting Information}

The Supporting Information is available free of charge on the ACS Publications website at DOI: 10.1021/acs.nanolett.7b02642.

Experimental details, including a description of synthesis and characterization of $\mathrm{ZnO} \mathrm{NCs}$ as well as stopped flow, optical, and NMR experiments; and computational details, including a comparison of $\mathrm{ZnO}$ and $\mathrm{ZnO}: e_{\mathrm{CB}}^{-} /$ $\mathrm{H}^{+}$structures, depiction of Morse potentials, illustration of delocalized nature of $\mathrm{ZnO}: e_{\mathrm{CB}}^{-} \mathrm{HOMO}$, analysis of dominant contributions to PCET rate constant, and optimized geometries. (PDF)

\section{AUTHOR INFORMATION}

\section{Corresponding Author}

*E-mail: shs3@illinois.edu.

\section{ORCID}

James M. Mayer: 0000-0002-3943-5250

Sharon Hammes-Schiffer: 0000-0002-3782-6995

\section{Notes}

The authors declare no competing financial interest.

\section{ACKNOWLEDGMENTS}

This work was supported as part of the Argonne-Northwestern Solar Energy Research (ANSER) Center, an Energy Frontier Research Center funded by the U.S. Department of Energy, Office of Science, Office of Basic Energy Sciences.

\section{REFERENCES}

(1) Klahr, B.; Gimenez, S.; Fabregat-Santiago, F.; Hamann, T.; Bisquert, J. J. Am. Chem. Soc. 2012, 134, 4294-4302.

(2) Mandal, H.; Shyamal, S.; Hajra, P.; Samanta, B.; Fageria, P.; Pande, S.; Bhattacharya, C. Electrochim. Acta 2014, 141, 294-301.

(3) Shaddad, M. N.; Ghanem, M. A.; Al-Mayouf, A. M.; Gimenez, S.; Bisquert, J.; Herraiz-Cardona, I. ChemSusChem 2016, 9, 2779-2783.

(4) Yao, T.; Chen, R.; Li, J.; Han, J.; Qin, W.; Wang, H.; Shi, J.; Fan,

F.; Li, C. J. Am. Chem. Soc. 2016, 138, 13664-13672.

(5) Wan, X.; Niu, F.; Su, J.; Guo, L. Phys. Chem. Chem. Phys. 2016, $18,31803-31810$.

(6) Sohn, Y.; Huang, W.; Taghipour, F. Appl. Surf. Sci. 2017, 396, 1696-1711.

(7) Jensen, S. C.; Bettis Homan, S.; Weiss, E. A. J. Am. Chem. Soc. 2016, 138, 1591-1600.

(8) Hagfeldt, A.; Graetzel, M. Chem. Rev. 1995, 95, 49-68.

(9) Zhao, Z.; Liu, H.; Chen, S. Nanoscale 2012, 4, 7301-7308.

(10) Mohamed, H. H.; Bahnemann, D. W. Appl. Catal., B 2012, 128, 91-104.

(11) Akimov, A. V.; Neukirch, A. J.; Prezhdo, O. V. Chem. Rev. 2013, $113,4496-4565$.

(12) Zhang, J.; Steigerwald, M.; Brus, L.; Friesner, R. A. Nano Lett. 2014, 14, 1785-1789.

(13) Chen, C.; Shi, T.; Chang, W.; Zhao, J. ChemCatChem 2015, 7, 724-731.

(14) Schrauben, J. N.; Hayoun, R.; Valdez, C. N.; Braten, M.; Fridley, L.; Mayer, J. M. Science 2012, 336, 1298-1301.

(15) Van de Walle, C. G. Phys. Rev. Lett. 2000, 85, 1012-1015.

(16) Wöll, C. Prog. Surf. Sci. 2007, 82, 55-120.

(17) Braten, M. N.; Gamelin, D. R.; Mayer, J. M. ACS Nano 2015, 9, $10258-10267$.

(18) Henkelman, G.; Uberuaga, B. P.; Jónsson, H. J. Chem. Phys. 2000, 113, 9901-9904.

(19) Perdew, J. P.; Burke, K.; Ernzerhof, M. Phys. Rev. Lett. 1996, 77, $3865-3868$.
(20) Zhang, Y.; Yang, W. Phys. Rev. Lett. 1998, 80, 890-890.

(21) Perdew, J. P.; Ruzsinszky, A.; Csonka, G. I.; Vydrov, O. A.; Scuseria, G. E.; Constantin, L. A.; Zhou, X.; Burke, K. Phys. Rev. Lett. 2008, 100, 136406.

(22) Meyer, B.; Marx, D. Phys. Rev. B: Condens. Matter Mater. Phys. 2003, 67, 035403.

(23) Guidon, M.; Hutter, J.; VandeVondele, J. J. Chem. Theory Comput. 2009, 5, 3010-3021.

(24) Guidon, M.; Hutter, J.; VandeVondele, J. J. Chem. Theory Comput. 2010, 6, 2348-2364.

(25) Valdez, C. N.; Delley, M. F. Private communication, 2017.

(26) Valdez, C. N.; Schimpf, A. M.; Gamelin, D. R.; Mayer, J. M. J. Am. Chem. Soc. 2016, 138, 1377-1385.

(27) Soudackov, A. V.; Hammes-Schiffer, S. J. Chem. Phys. 2000, 113, 2385-2396.

(28) Soudackov, A. V.; Hatcher, E.; Hammes-Schiffer, S. J. Chem. Phys. 2005, 122, 014505 .

(29) Venkataraman, C.; Soudackov, A. V.; Hammes-Schiffer, S. J. Phys. Chem. C 2008, 112, 12386-12397.

(30) Hammes-Schiffer, S.; Hatcher, E.; Ishikita, H.; Skone, J. H.; Soudackov, A. V. Coord. Chem. Rev. 2008, 252, 384-394.

(31) Marcus, R. A. Annu. Rev. Phys. Chem. 1964, 15, 155-196.

(32) Marcus, R. A. J. Chem. Phys. 1965, 43, 679-701.

(33) Kuznetsov, A. M.; Ulstrup, J. Electron Transfer in Chemistry and Biology: An Introduction to the Theory; Wiley: Hoboken, NJ, 1999.

(34) Cohn, A. W.; Janßen, N.; Mayer, J. M.; Gamelin, D. R. J. Phys. Chem. C 2012, 116, 20633-20642.

(35) Kondov, I.; Čížek, M.; Benesch, C.; Wang, H.; Thoss, M. J. Phys. Chem. C 2007, 111, 11970-11981.

(36) Warren, J. J.; Tronic, T. A.; Mayer, J. M. Chem. Rev. 2010, 110, 6961-7001.

(37) Gratzel, M. Nature 2001, 414, 338-344.

(38) Lemon, B. I.; Hupp, J. T. J. Phys. Chem. B 1997, 101, 24262429.

(39) Mayer, J. M. Manuscript in preparation; to be submitted to J. Am. Chem. Soc. in 2018.

(40) Klimkans, A.; Larsson, S. Chem. Phys. 1994, 189, 25-31.

(41) Fernandez, L. E.; Horvath, S.; Hammes-Schiffer, S. J. Phys. Chem. C 2012, 116, 3171-3180.

(42) Valdez, C. N.; Schimpf, A. N.; Gamelin, D. R.; Mayer, J. M. ACS Nano 2014, 8, 9463-9470.

(43) Eberson, L. Adv. Phys. Org. Chem. 1982, 18, 79-185. 